\title{
MEMBRANAS ANTIMICROBIANAS BIOPOLIMÉRICAS OBTIDAS POR POLICOMPLEXAÇÃO PARA O TRATAMENTO E CICATRIZAÇÃO DE FERIDAS
}

\author{
F. S. GUIRAU ${ }^{1}$, M. R. PIRES ${ }^{1}$ e S. V. G. NISTA ${ }^{1}$ \\ ${ }^{1}$ Faculdade de Americana (FAM), Departamento de Engenharia Química. \\ E-mail para contato: felipe.guirau3@hotmail.com
}

\begin{abstract}
RESUMO - O objetivo deste trabalho foi o desenvolvimento de uma membrana de biopolímeros com a incorporação de eugenol com propriedades antimicrobianas, que poderá ser utilizado como auxiliar em cicatrização de feridas. O recobrimento de ferida ideal deve substituir as funções da pele perdida e o material deve ser biocompatível para evitar rejeição e apresentar um custo acessível para que sua utilização seja economicamente viável. As membranas foram preparadas pelo método layer-by-layer utilizando os biopolímeros quitosana e alginato. Foi realizada a policomplexação dos biopolímeros entre as camadas, com o objetivo de reduzir sua solubilidade em meio biológico. $\mathrm{O}$ principio ativo foi incorporado na membrana e o perfil de liberação do mesmo in vitro foi determinado. Foi verificada a viabilidade na preparação da membrana.
\end{abstract}

\section{INTRODUÇÃO}

O eugenol é um químico aromático muito versátil, o qual vem sendo empregado por vários campos da indústria. Apresenta um importante efeito antibacteriano e antifúngico, pois mostra se ativo contra Escherichia coli, Streptococcus pneumoniae, Staphylococcus aureus, Candida albicans dentre outros agentes patogênicos (Azambuja, 2015). Além de poderoso antisséptico apresenta efeitos anti-inflamatório, cicatrizante, analgésico e é eficaz como parasiticida e antimicótico, com ação antiespasmódica e anti-histamínica (Revilla, 2002).

Sistemas poliméricos para liberação controlada de fármacos têm inúmeras vantagens, comparadas às formas normais de dosagem. Nestes sistemas, os níveis de fármacos no plasma são continuamente mantidos em uma faixa terapêutica desejável, sendo que os efeitos colaterais nocivos observados na administração convencional podem ser reduzidos, ou eliminados, pela administração local (Zeng et al., 2003).

Historicamente, o tratamento de feridas tem como objetivo a proteção das lesões contra a ação de agentes externos físicos, mecânicos ou biológicos. É um meio terapêutico que consiste na aplicação de uma cobertura sobre uma ferida limpa. Até o século 20, os recobrimentos de feridas eram considerados produtos passivos, com mínima influência no processo de cicatrização. $\mathrm{O}$ conceito de incorporação de fármacos em uma membrana, para utilização como recobrimentos para feridas, melhorando a eficiência destes materiais, iniciouse com a pesquisa pioneira de Winter, na década de 60, onde foi considerado o envolvimento ativo do recobrimento com a ferida, com a finalidade de estabelecer e manter um ambiente ótimo para a cicatrização da mesma. (Chen et al., 2007). Pesquisas desenvolvidas na área biomédica geraram várias opções comerciais de recobrimentos de feridas, entretanto tais produtos apresentam alto custo, o que dificulta a aplicação e comercialização dos mesmos em países emergentes. 
$\mathrm{Na}$ área de bioengenharia, a formação de polieletrólito tem sido muito explorada na produção de vários sistemas de liberação controlada de fármacos, à base de polissacarídeos, para aplicação em diversos locais do corpo humano (Goycoolea et al., 2009), devido às várias propriedades interessantes, como mucoadesão e bioadesividade. Este sistema tem uma elevada capacidade de se associar e liberar macromoléculas terapêuticas na sua forma bioativa, bem como melhorar o transporte de compostos bioativos através das bem organizadas barreiras epiteliais (Goycoolea et al., 2009).

Polieletrólitos são macromoléculas que tem um papel importante no transporte de um número relativamente grande de grupos funcionais carregados. Eles podem ter a função de policátions ou poliânions, uma vez que a carga líquida destas macromoléculas em solução depende dos grupos funcionais, que podem estar carregados positiva ou negativamente, dependendo do $\mathrm{pH}$ do meio (Nista et al., 2015).

A quitosana e o alginato são polieletrólitos bem conhecidos, biocompatíveis, biodegradáveis e mucoadesivos, permitindo várias aplicações farmacêuticas e biomédicas (Nista et al., 2015). Podem ser utilizados como agentes espessantes, na indústria alimentícia; como sistemas de liberação de fármaco, em aplicações farmacêuticas, e como biomateriais em cultura de células e cicatrização de feridas. Estes dois polissacarídeos também podem ser usados em conjunto, formando um complexo polieletrolítico, muito utilizado para encapsular proteínas, células e enzimas (Nista et al., 2015).

A quitosana é a forma acetilada da quitina, mostra unidades acetiladas (2-acetoamido2deoxi-D-glicopiranose) e desacetiladas (2-amino-deoxi-D-glicopiranose), unidas por ligações glicosídicas, com predominância das unidades desacetiladas (Kleinubing et al., 2013). A quitosana é normalmente extraída de casca de crustáceos como camarão, caranguejo e lagosta. Ela tem sido investigada como veículo de entrega de fármaco, porque é capaz de reduzir a resistência elétrica transepitelial.

O alginato de sódio é extraído principalmente de 3 espécies de algas, ou seja, a Macrocystis pyrifera, a Laminaria hyperborea e a Ascophyllum nodosum. Quimicamente é um biopolímero aniônico, composto de cadeias lineares de ácido $\beta$-D-manurônico (unidade M) e do ácido $\alpha$-L-gulurônico (unidade G) (Kleinubing et al., 2013). Ele é um dos polieletrólitos aniônicos mais estudados na complexação com a quitosana, porque o policomplexo formado entre estes dois polímeros ainda é biodegradável, biocompatível e mecanicamente mais resistente (Hamman et al., 2010). A formação do complexo polieletrolítico é facilitada por um mecanismo eletrostático, onde a neutralização de cargas ocorre por interações tipo ligações de hidrogênio, forças de Coulomb e forças de Van der Waals, conforme mostra a Figura 1.

Uma grande vantagem do complexo polieletrolítico quitosana-alginato é sua preparação que ocorre em água, dispensando o uso de solventes orgânicos tóxicos, no preparo de membranas de revestimento para sistemas de liberação controlada. As membranas assim obtidas são biodegradáveis e insolúveis em água, com grande potencial para aplicações biomédicas (Meng et al., 2010). 
Figura 1 - Interações iônicas entre o alginato de sódio e a quitosana (Knill et al., 2004).

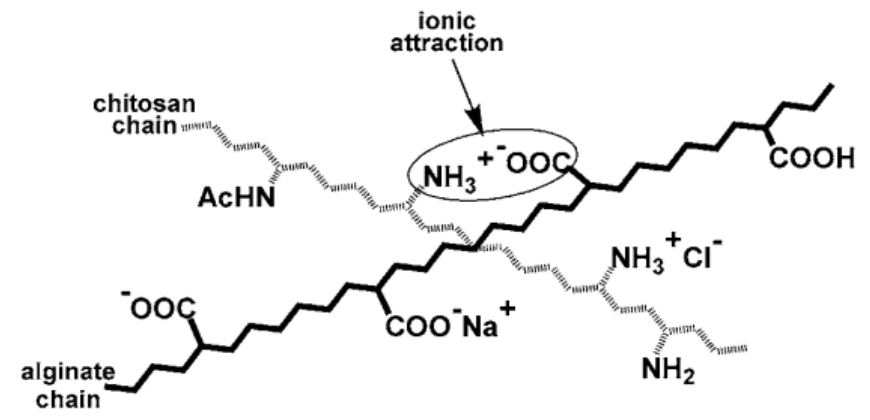

O objetivo deste trabalho é o desenvolvimento de uma membrana de biopolímeros com a incorporação de eugenol com propriedades antimicrobianas, que poderá ser utilizado como auxiliar em cicatrização de feridas. Com o que foi apresentado nesta introdução acredita-se que o dispositivo proposto apresenta-se como uma alternativa viável e de baixo custo como recobrimento ativo de lesões da pele.

\section{MATERIAIS E MÉTODOS}

Inicialmente foi preparada uma solução de $1,5 \%$ de quitosana $(\mathrm{m} / \mathrm{m})$ em solução ácido acético $1 \mathrm{M}$ e mantida em agitação até completa solubilização. A solução de Alginato foi preparada através de uma solução de $2 \%$ de alginato $(\mathrm{m} / \mathrm{m})$ em água destilada com adição de $20 \%$ (m/m base polímero) de eugenol e mantida em agitação até completa solubilização.

A produção da membrana composta de quitosana e alginato foi baseada no método layer-by-layer de produção de membranas multicamadas. Iniciou-se com o espalhamento de $12 \mathrm{ml}$ da solução de quitosana sobre uma placa de Petri de $9 \mathrm{~cm}$ de diâmentro. Esta foi colocada em estufa a $50^{\circ} \mathrm{C}$ para evaporação do solvente. Em seguida, foi espalhada a solução de alginato com eugenol sobre a camada de quitosana seca, também em quantidade suficiente para se cobrir a placa com uma fina camada de solução, como anteriormente. A placa foi novamente levada à estufa para evaporação do solvente, e para finalizar foi realizada a deposição de mais uma camada de quitosana, $12 \mathrm{ml}$ da solução na placa.

A quantificação do eugenol foi realizada através da construção da curva de calibração, onde soluções de eugenol em concentrações conhecidas foram preparadas em solução de soro fisiológico/etanol (1:1) e a determinação da absorbância no $\lambda 283 \mathrm{~nm}$ em espectrômetro UVVis para cada solução foi determinada. Foi construída uma curva na faixa de 1 a $50 \mu \mathrm{g} / \mathrm{ml}$. Para cada solução, de concentração conhecida, foram retiradas três amostras para leitura da absorbância.

O estudo da liberação do óleo eugenol das membranas foi determinado através de uma análise onde as amostras da membrana, com massa conhecida, foram colocadas em tubos de ensaio e alíquotas de $10 \mathrm{ml}$ de solução soro fisiológico/etanol (1:1) foram adicionadas em cada tubo, os quais foram deixados em banho termostático com temperatura controlada a $37^{\circ} \mathrm{C}$. Em períodos pré-determinados, alíquotas de $1 \mathrm{ml}$ da solução foram retiradas para análise quantitativa de eugenol e substituídas por uma nova alíquota de soro fisiológico/etanol (1:1). O tempo total de análise no teste de liberação foi de 5 horas. As alíquotas foram analisadas no comprimento de onda máxima do óleo eugenol, $\lambda$ máx $283 \mathrm{~nm}$. A quantidade de 
eugenol liberado com o tempo foi determinada usando a curva de calibração do óleo construída, que relaciona a absorbância com a concentração conhecida do eugenol.

\section{RESULTADOS E DISCUSSÕES}

A formação da membrana layer-by-layer de quitosana-alginato se deu através da interação eletrostática entre as cadeias poliméricas: a quitosana se comporta como um policátion, devido aos grupos amino, e o alginato se comportam como um poliânion, devido aos grupos hidroxila presentes. Na Figura 2 pode ser verifica a membrana obtida.

Figura 2 - Amostra da membrana layer by layer quitosana/alginato em solução soro fisiológico/etanol (1:1)

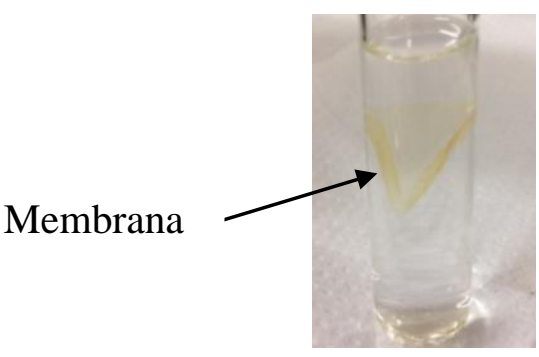

Com o auxílio de um espectrofotômetro UV-Vis, e através de soluções de eugenol com concentrações conhecidas, foi possível determinar uma curva de calibração para o composto relacionando absorbância a $\lambda$ máx $283 \mathrm{~nm}$ com as concentrações, conforme mostra a Figura 3.

Figura 3- Curva de calibração do eugenol

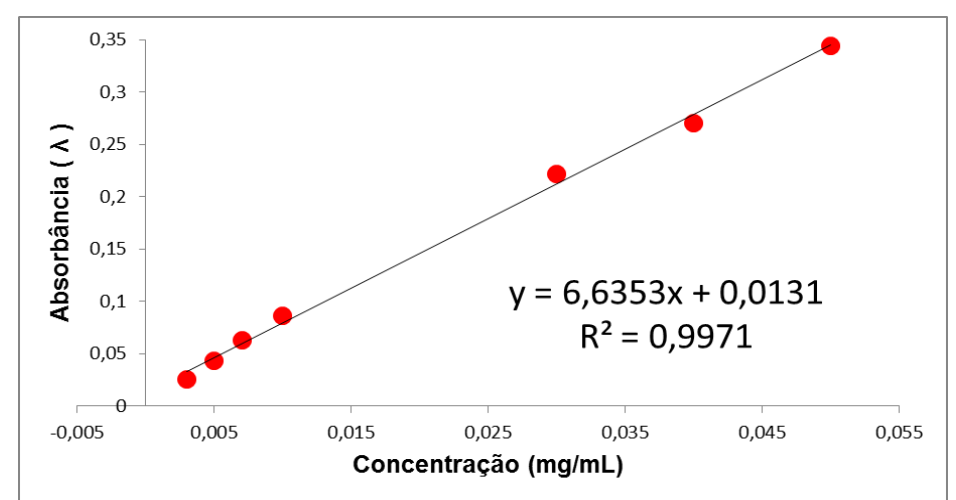

A quantidade de eugenol liberado em um determinado período de tempo foi determinada, quando submetido a um meio semelhante ao de uma lesão cutânea, que no caso, foi utilizado uma solução de soro fisiológico e etanol (1:1).

O teste foi realizado em um período total de 5 horas, com 9 intervalos para coleta de amostras, que foi feito em triplicata. Após as 5 horas, a amostra se mostrou com um leve intumescimento, indicando assim que houve liberação. Utilizando a curva de calibração como referência, foi possível determinar a concentração liberada de eugenol de cada amostra por período de tempo, que foram discriminados nos resultados da Tabela 1. 


\section{Congresso Brasileiro de Engenharia Química \\ em Iniciação Científica \\ UFSCar - São Carlos - SP

A Figura 4 mostra o perfil de liberação do Eugenol com o tempo. O perfil de liberação do princípio ativo na membrana preparada é lento, com liberação de apenas 3,65\% do total incorporado em 5 horas. Temos uma liberação inicial (burst release) de 2,5\% em 15 minutos, que é relativamente alta quando comparada ao total liberado.

Tabela 1 - Liberação do óleo eugenol em relação ao tempo

\begin{tabular}{|c|c|c|}
\hline Tempo $(\mathbf{m i n})$ & Média liberação $(\boldsymbol{\mu g})$ & Média liberação $(\boldsymbol{\%})$ \\
\hline \hline 15 & 55,11 & 2,6 \\
\hline 30 & 55,51 & 2,6 \\
\hline 45 & 63,88 & 3,0 \\
\hline 60 & 66,93 & 3,2 \\
\hline 90 & 67,13 & 3,2 \\
\hline 120 & 67,73 & 3,2 \\
\hline 180 & 72,18 & 3,4 \\
\hline 240 & 72,78 & 3,4 \\
\hline 300 & 77,44 & 3,6 \\
\hline
\end{tabular}

Figura 4 - Curva de liberação de eugenol.

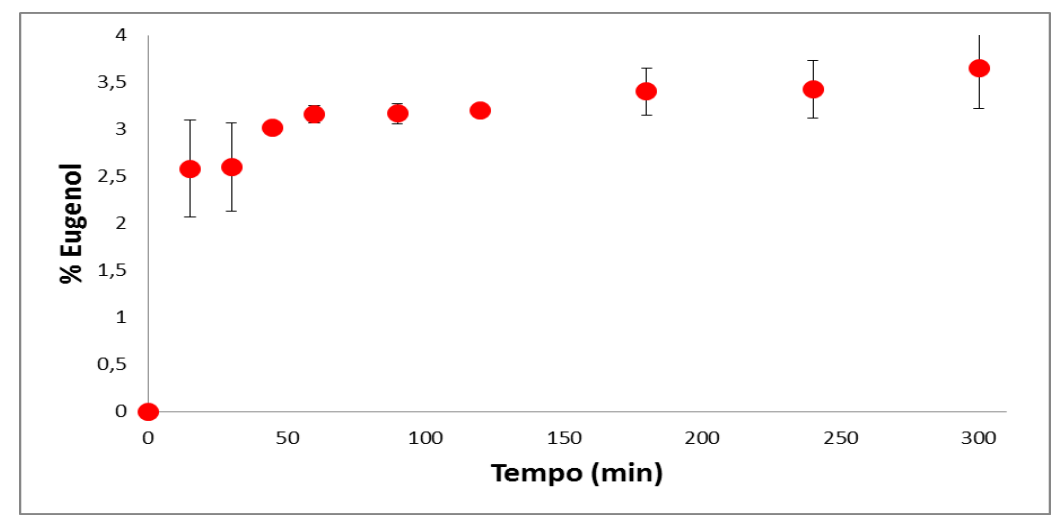

Conforme Martins (2012), o eugenol é ativo contra Escherichia coli e Staphylococcus aureus, que são as bactérias mais encontradas em lesões de pele, justificando assim, a escolha do eugenol como agente antimicrobiano. Foi obtida neste mesmo trabalho a inibição de $E$. coli com concentração superior a $35 \%$ de eugenol e de $S$. aureus com uma concentração superior a 50\% de eugenol na amostra. Martins (2012) utilizou uma membrana de acetato de celulose, onde o perfil de liberação é diferente. O acetato de celulose é hidrofóbico e a quitosana é hidrofílica. Na amostra de quitosana, que é hidrofílica, o eugenol que é hidrofóbico, tende a ser liberado mais rapidamente, pois prefere o meio (etanol/soro). No caso do acetato de celulose que é hidrofóbico ele tende a ser mais retido pela membrana. Dessa forma, acredita-se que será necessário um aumento na concentração incorporada de eugenol na amostra para obter o efeito antimicrobiano desejado.

Segundo Pereira (2007), onde também foi preparada uma membrana $100 \%$ de quitosana, mas por outra técnica, spray dry, foi obtido uma liberação de $50 \%$ do eugenol em apenas 7 minutos de liberação. No método por camadas, a liberação mostrou um perfil mais lento e, assim, mais adequado para liberação prolongada de princípios ativos. Este efeito 
provavelmente se deve ao processo de preparação da membrana pela técnica de layer by layer por "casting" e pelo processo de reticulação por policomplexação do material.

\section{CONCLUSÃO}

Foi possível verificar a viabilidade da preparação da membrana proposta bem como o perfil de liberação do Eugenol no material. Este trabalho está em andamento e outros testes serão realizados aumentando-se a concentração de eugenol na amostra, bem como testes microbiológicos com as bactérias Escherichia coli e Staphylococcus aureus para verificar a ação efetiva da membrana desenvolvida.

\section{REFERÊNCIAS}

AZAMBUJA, W. Eugenol. Óleos Essenciais, 2015. Disponível em: <http://www.oleosessenciais.org/eugenol/>. Acesso em: 13/03/2017.

CHEN, C.; WANG, L.; HUANG, Y. Electrospinning of thermo-regulating ultrafine fibers based on polyethyleneglycol/cellulose acetate composite. Polymer 2007, 48, 5202-5207.

GOYCOOLEA, F. M., LOLlO, J., REMUÑAN-LOPEZ, C., QUAGLIA, F., \& ALONSO, M. J. Chitosan-alginate blended nanoparticles as carriers for the transmucosal delivery of macromolecules. Biomacromolecules 2009, 10, 17361743.

HAMMAN, J. H. Chitosan based polyelectrolyte complexes as potential carrier materials in drug delivery systems. Marine Drugs 2010, 8, 1305-1322.

KLEINUBING, S. A., SERAPHIM, D. C., VIEIRA, M. G. A., CANEVESI, R. L. S., SILVA, E. A., CÉSAR, C. L. Gastro-resistant controlled release of OTC encapsulated in alginate/chitosan matrix coated with acryl-EZE ${ }^{\circledR} M P$ in fluidized bed. J. App. Polym. Sci. 2014, 131, 40444.

MARTINS, Renata; Atividade antimicrobiana de óleos essenciais e incorporação em filme de acetato celulose na conservação de carne resfriada de frango. UFG, Goiânia, 2012.

MENG, X., TIAN, F., YANG, J., HE, C. N., XING, N., \& LI, F. Chitosan and alginate polyelectrolyte complex membranes and their properties for wound dressing application. J. Mat. Sci: Mater in Medicine 2010, 21, 17511759.

NISTA S V G, BETTINI J, MEI, L H I. Coaxial nanofibers of chitosan - alginate - PEO polycomplex obtained by electrospinning, Carbohydr Polym. 2015, 127, 222.

PEREIRA, Taize. Microencapsulação do óleo eugenol pelo método de Spray Dryng. UFSC, Florianópolis, 2006.

REVILLA, Juan. Apontamentos para a cosmética Amazônica. Pág. 260; 2002.

Sec. Saúde Prefeitura Municipal de Ribeirão Preto, Manual Assistência Integral as pessoas com Feridas Crônicas, 2004, Ribeirão Preto.

ZENG, J.; XU, X.; CHEN, X.; LIANG, Q.; BIAN, X.; YANG, L.; JING, X. Biodegradable electrospun fibers for drug delivery. J. Control. Release 2003, 92, 227-231. 